\title{
Detection of multiple modes in a new double-mode RR Lyrae star ${ }^{\star}$ (Research Note)
}

\author{
M. Chadid \\ Université Nice Sophia-Antipolis, Observatoire de la Côte d'Azur, UMR 7293, Parc Valrose, 06108 Nice Cedex 02, France \\ Antarctica Reaserch Station, South Pole, TAAF, Antarctica \\ e-mail: chadid@unice.fr \\ Received 4 June 2011 / Accepted 7 February 2012 \\ ABSTRACT \\ Context. We present the first long-time coverage of high precision and uninterrupted light curve of a new double-mode RR Lyrae star \\ allowing the determination of accurate Fourier components and the estimatation of fundamental parameters. \\ Aims. We used high-precision space data of a new star CoRoT ID 0101368812, obtained by the satellite CoRoT, towards an under- \\ standing of double-mode RR Lyrae stars and the stellar evolution \& pulsation. \\ Methods. The CoRoT data were corrected using jump and trend filtering codes and we applied the period-finding technique Period04. \\ Results. Non-linearly coupled frequencies $f_{0}=2.049 \mathrm{~d}^{-1}\left(P_{0}=0.488 \mathrm{~d}\right)$ and $f_{1}=2.750 \mathrm{~d}^{-1}\left(P_{1}=0.364 \mathrm{~d}\right)$ are found up to the tenth \\ order with a period ratio $P_{1} / P_{0}$ of 0.745 and an amplitude ratio $A_{1} / A_{0}$ of 2.705 . The frequencies $f_{0}$ and $f_{1}$ are interpreted as radial \\ modes. The first overtone is the dominant pulsation mode. A large number of 22 linear combinations of these modes are also detected. \\ An unprecedented number of additional frequencies and their linear combinations, are detected in addition to the fundamental radial \\ mode, the first overtone radial mode, and their linear combinations. The most surprising result is the unexpected period ratios 0.458 , \\ 0.285 , and 0.217 of additional frequencies to fundamental radial mode, which imply a possible simultaneous excitation of fundamen- \\ tal, first, and third overtone radial modes, in addition to non-radial modes. \\ Conclusions. The newly discovered CoRoT ID 0101368812 is the first member of a new class of RR Lyrae stars. These results give \\ us an opportunity to test the theoretical models of mode saturation and growth in RR d pulsators.
}

Key words. hydrodynamics - shock waves - techniques: photometric - stars: atmospheres - stars: variables: RR Lyrae stars: Population II

\section{Introduction}

Double-mode RR Lyrae stars, namely RR d, pulsate simultaneously in the first overtone and fundamental radial modes (Nemec 1984). Their period ratio ranges from about 0.74 to 0.75 and the first overtone radial mode is usually dominant (Oaster et al. 2006).

The RR d stars play a key role in the mass determinations based on the period ratio independently of stellar evolution theory (Smith 1995). They have been identified in the Galactic field, the Magellanic Clouds, globular clusters, and dwarf spheroidal galaxies (Clement et al. 1991). The RR d stars have been studied spectroscopically and photometrically by Clement et al. (1991), Clementini et al. (2000), and Dekany et al. (2008), focusing on AQ Leo, CU Com, and BS Com.

Using MOST optical photometry of the RR d star AQ Leo, Gruberbauer et al. (2007) reported that AQ Leo $\left(P_{1}=0.410 \mathrm{~d}\right.$, $P_{0}=0.550 \mathrm{~d}$, and $P_{1} / P_{0}$ of 0.745$)$ oscillates with additional frequencies besides the fundamental radial mode, the first overtone radial mode and their linear combinations. They suggested that there are two possibly additional non-radial pulsation modes. Dekany et al. (2008) derived the basic physical parameters of RRd star BS Com $\left(P_{1}=0.363 \mathrm{~d}, P_{0}=0.488 \mathrm{~d}\right.$, and $P_{1} / P_{0}$ of 0.744 ) by using evolutionary models and ground-based photometric observations. They did not detect additional frequencies in the BS Com Fourier spectrum.

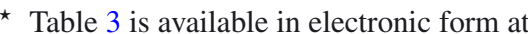
http: //www . aanda.org
The nature of the additional frequencies in RR d stars, as well as in Blazhko stars (Chadid et al. 2010), needs further investigation. The continuous and unprecedented CoRoT photometric data provide a unique opportunity to obtain an RR d light curve over relatively long time period (150 days) and with a relatively high precision.

In this paper, we discuss the discovery of a new doublemode RR Lyrae star, CoRoT ID 0101368812, which exhibits additional frequency terms. The star CoRoT ID 0101368812 $(\alpha=192813.645, \delta=+004042.83, V=15.60 \mathrm{mag})$ was observed during the first long run in the center direction of the Galaxy LRc01, from May 16th to October 15th, 2007.

Section 2 describes the CoRoT data reduction. Section 3 is devoted to the description of data analysis with our findings. The fundamental parameters are presented in Sect. 4. Finally, some concluding remarks are given in Sect. 5.

\section{Observations and data reduction}

We used the CoRoT data of the target CoRoT ID 0101368812, which is a new RR Lyrae star observed during the first long run targeting the Galactic center (LRc01). The CoRoT data are the calibrated light curves, N2-level, and we used here the resulting "white" flux. The total time span is 152 days (Fig. 1). The time sampling of the light curves is $512 \mathrm{~s}$, giving a time resolution of $1.2 \%$ of the pulsation fundamental period. The CoRoT $\mathrm{N} 2$ data are affected by jumps, trends, and a long-term drift in the light curves owing to changes in the amount of incident stray 

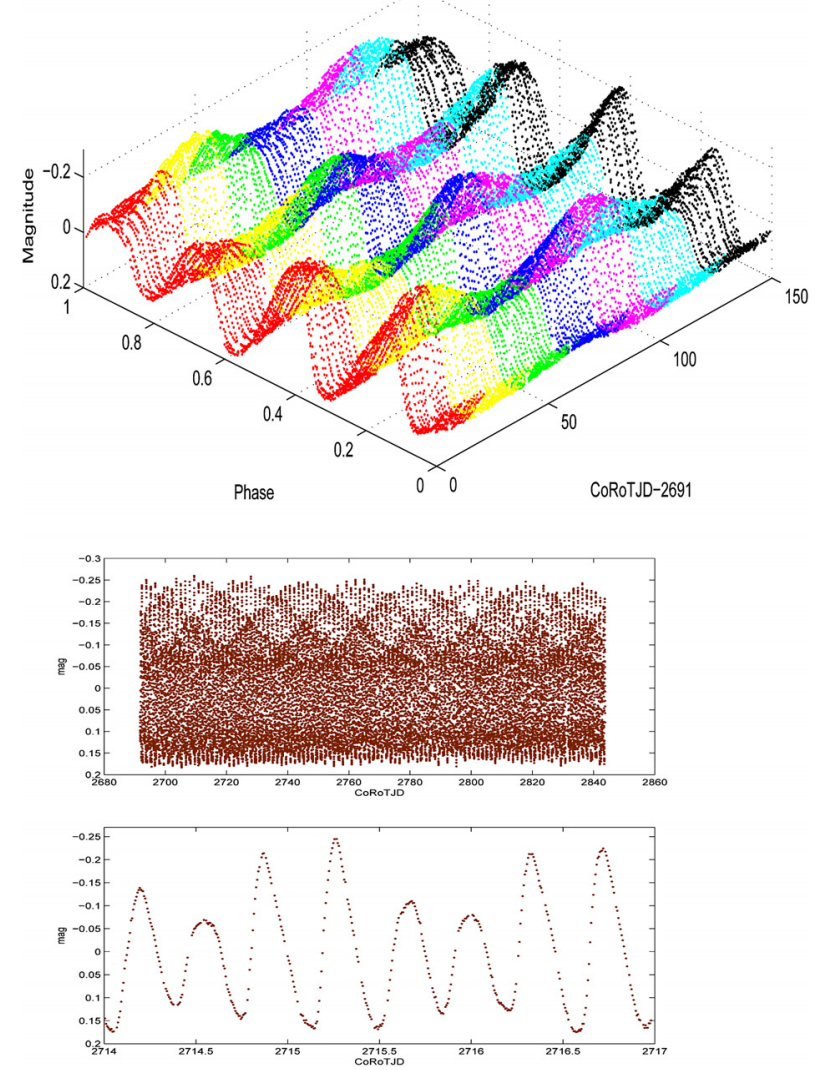

Fig. 1. Top panel: two-dimensional data folded with the period $1.426 \mathrm{~d}$. Middle panel: the entire time series. Bottom panel: an expanded view of the light curve.

light during the run, periodic changes in flux caused by the satellite orbit, and discontinuities in the light curves caused by cosmic ray hits on the CCDs. Thus, a trend-and-jump filtering code was applied to the data. The trend-filtering process divides the data set into bins, and within each bin the fluxes and times are averaged. The independently binned, average values of fluxes are interpolated between the original points of time and subtracted from the observed ones. The jump filtering process is based on the difference between these values and the subsequent points of the light curve. A difference is regarded as a jump if it exceeds a certain faraction of the standard deviation (Chadid et al. 2010).

\section{Frequency analysis}

The frequency analysis was performed using Period04 (Lenz \& Breger 2005). The Fourier decomposition consists of fitting the magnitude measurements by means of the series

$m(t)=A_{0}+\sum_{i=1, N} A_{i} \sin \left[2 \pi\left(F_{i}\left(t-T_{0}\right)+\phi_{i}\right)\right]$,

where $T_{0}=2691.75315$ is the initial epoch value of the data set CoRoTJD. To check our frequency solution, we ran the program package SigSpec, which calculates the spectral significance sig defined by Reegen (2007) for each peak of the spectrum. Fortynine significant frequencies are clearly detected with a threshold value of sig = 5 (Table 3 ). However, the regular behavior of harmonics in RR Lyrae stars helps us to identify frequency peaks, which are intrinsic to the star's pulsation, of a lower amplitude than the accepted value. By means of a rigorous check of the high-frequency region, where the noise level is lower, we were able to detect the harmonics below the generally accepted significance level. Our data set for CoRoT ID 0101368812 consists of 415 consecutive pulsation cycles (Fig. 1).

\subsection{Fundamental and first overtone radial modes}

Figure 3 shows the amplitude spectrum of CoRoT ID 0101368812. We clearly observe two dominant frequencies $f_{0}=$ $2.049009 \pm 0.000005 \mathrm{~d}^{-1}\left(P_{0}=0.4880408 \pm 0.0000001 \mathrm{~d}\right)$, and $f_{1}=2.750263 \pm 0.000002 \mathrm{~d}^{-1}\left(P_{1}=0.3636016 \pm 0.0000003 \mathrm{~d}\right)$. The period ratio $P_{1} / P_{0}=0.7450229$ corresponds to the period ratio of RRd in the Petersen diagram (Petersen 1973). We detect the harmonics of the fundamental radial mode $f_{0}$ up to third order and those of the first overtone radial mode $f_{1}$ up to tenth order. The amplitude ratio $A_{1} / A_{0}$ of 2.7054968 shows that the first overtone is the dominant pulsation mode and characterized by stronger non-linear behaviour which can be seen by the more pronounced non-sinusoidal shape of its light curve in Fig. 2.

Twenty-two linear combinations of frequency components of the fundamental and first overtone modes are detected. Their amplitudes are smaller than those of their constituent frequencies. The frequency terms of the positive linear combinations have larger amplitudes than those of the negative linear combinations. This seems to agree with the double-mode Cepheid models (Antonello \& Aikawa 1998).

\subsection{Additional frequencies}

A detailed analysis reveals that the power spectrum has a complex structure in the frequency domain around $f_{0}$ and $f_{1}$. After prewhitening of the dominant radial frequency $f_{1}$ and its harmonics, we see many additional peaks in the Fourier spectrum. Figure 3 shows the residual spectrum after prewhitening of the dominant frequency $f_{1}$ and its harmonics. We clearly detect three independent frequencies:

- $f_{2}=4.479 \mathrm{~d}^{-1}:$ The frequency $f_{2}=4.4786159 \pm$ $0.000002 \mathrm{~d}^{-1}\left(P_{2}=0.2232832 \pm 0.0000004 \mathrm{~d}\right)$ has an amplitude that is smaller than $3 f_{1}$ and at the same level as that of the $2 f_{0}$ harmonic. It occurs between $f_{1}$ and its first harmonic and appears at a frequency ratio $f_{1} / f_{2}$ of 0.6140875 . The frequency $f_{2}$ has a period ratio $P_{2} / P_{0}$ of 0.4575094 and an amplitude ration $A_{2} / A_{0}$ of 0.1044413 .

- $f_{3}=7.194 \mathrm{~d}^{-1}$ : Occurring between second and third harmonics of $f_{1}$, the frequency $f_{3}=7.1943909 \pm 0.000002 \mathrm{~d}^{-1}$ $\left(P_{3}=0.1389971 \pm 0.0000004 \mathrm{~d}\right)$ has smaller amplitude than the $4 f_{1}$ and $2 f_{0}$ harmonics, with a frequency ratio $f_{1} / f_{3}$ of 0.3822782 . We measure a period ratio $P_{3} / P_{0}$ of 0.2848064 and an amplitude ratio of $A_{3} / A_{0}$ of 0.0319339 .

- $f_{4}=9.433 \mathrm{~d}^{-1}$ : The amplitude of $f_{4}=9.4325280 \pm$ $0.000002 \mathrm{~d}^{-1}\left(P_{4}=0.1060161 \pm 0.0000006 \mathrm{~d}\right)$ is smaller than $2 f_{0}$ and at the same level of that of the $7 f_{1}$ harmonic. It appears between the third and fourth harmonics of the dominant mode $f_{1}$, showing a frequency ratio $f_{1} / f_{4}$ of 0.2915721 , a period ratio $P_{4} / P_{0}$ of 0.2172279 , and an amplitude ration $A_{4} / A_{0}$ of 0.0151979 .

Table 1 gives the relevant values of period and amplitude ratios. The frequencies $f_{2}, f_{3}$, and $f_{4}$ consist of linear combinations of the fundamental and the first overtone radial modes and their harmonics. All the detected coupling terms of the positive linear combinations have larger amplitudes than those of the negative linear combinations (see Table 3). 

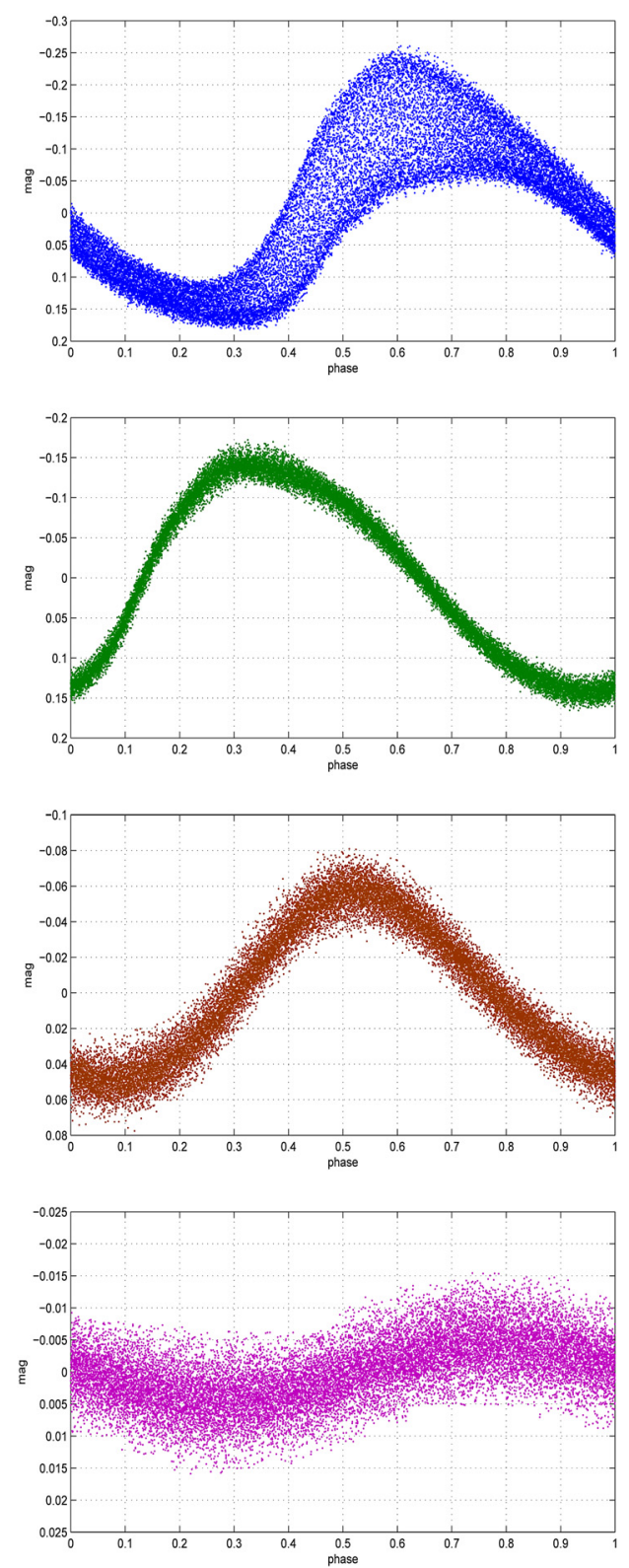

Fig. 2. Folded light curve of CoRoT ID 0101368812. From the upper to lower panel: a) folded with $P_{1}$; b) prewhitened with $P_{0}$ and folded with $\left.P_{1} ; \mathbf{c}\right)$ prewhitened with $P_{1}$ and folded with $\left.P_{0} ; \mathbf{d}\right)$ folded with $P_{2}$ after removal of $P_{0}$, and $P_{1}$, combination frequencies, and the additional frequencies $P_{3}$ and $P_{4}$.

The origin of the additional frequencies $f_{2}, f_{3}$, and $f_{4}$ is a great challenge. Judging from the period ratio alone, the ratio $P_{2} / P_{0}=0.458$ is very close to that of the third overtone to fundamental radial mode. Thus the frequency $f_{2}=4.479 \mathrm{~d}^{-1}$ is likely related to the third radial overtone mode. A similar case was reported for the star AQ Leo, for which a frequency $f_{i i}=3.92593 \mathrm{~d}^{-1}$ was detected by the MOST satellite (Gruberbauer et al. 2007): this implies a ratio to the fundamental radial mode $(1.81896 / 3.92593=0.463)$, which is perhaps related to the third radial overtone mode. This strengthens our argument for the detection reliability of the third radial-overtone mode pulsation. On the other hand, the period ratio of $f_{2}$ to the dominant first radial overtone mode $f_{1}$ is 0.614 . Such an unexpected period ratio of 0.61 was reported for RR Lyrae variables in the globular clusters $\omega$ Cen, V 19, and V 105 (Olech \& Moskalik 2009) and in
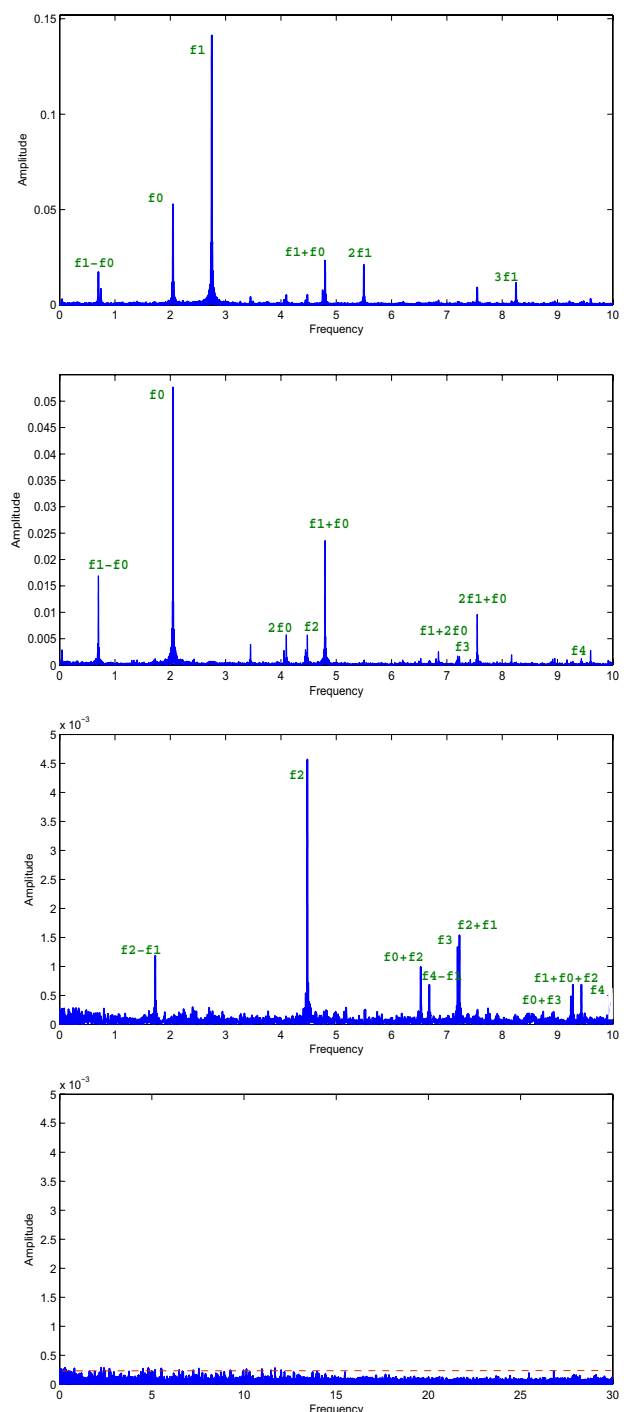

Fig. 3. From the upper to lower panels: a) the amplitude spectrum of the CoRoT ID 0101368812 light curve; b) the amplitude spectrum after prewhitening of the first overtone radial frequency and its harmonics; c) after prewhitening of the first overtone and the fundamental radial frequencies and their harmonics and their linear combination terms; and d) the residuals after prewhitening with all frequencies in Table 3. The dashed curve shows the noise level that corresponds to the level of the weakest amplitude in the data.

the LMC first overtone Cepheids (Moskalik \& Kolaczkowski 2008; Soszynski et al. 2008). Both cases were theoretically interpreted as non-radial modes (Dziembowski \& Smolec 2009). Hence, $f_{2}=4.479 \mathrm{~d}^{-1}$ may also be interpreted as a non-radial mode.

The additional frequencies $f_{3}\left(f_{3}-7 / 2 f_{0}=0.0225 \mathrm{~d}^{-1}\right)$ may be the seventh half-integer frequency of the fundamental radial mode $f_{0}$. Following this hypothesis, the presence of a half-integer frequency would be a clear sign of a period-doubling bifurcation in CoRoT ID 0101368812, as reported by Buchler \& Moskalik (1992) in Pop II Cepheids. The analysis of the limit cycle stability (Moskalik \& Buchler 1990) showed that 5:2 resonances, between the fundamental mode and a high-order radial overtone, are responsible for this instability in Pop II, that there is a coincidence between the period doubling and the resonance center, and then every half-integer resonance is able to cause period doubling. 
Table 1. Summary of the relevant data for CoRoT ID 0101368812.

\begin{tabular}{ll}
\hline \hline$P_{0}$ (days) & $0.4880408 \pm 0.0000001$ \\
$P_{1}$ (days) & $0.3636016 \pm 0.0000003$ \\
$P_{2}$ (days) & $0.2232832 \pm 0.0000004$ \\
$P_{3}$ (days) & $0.1389971 \pm 0.0000004$ \\
$P_{4}$ (days) & $0.1060161 \pm 0.0000006$ \\
$P_{1} / P_{0}-A_{1} / A_{0}$ & $0.7450229-2.7054968$ \\
$P_{2} / P_{0}-A_{2} / A_{0}$ & $0.4575094-0.1044413$ \\
$P_{3} / P_{0}-A_{3} / A_{0}$ & $0.2848064-0.0319339$ \\
$P_{4} / P_{0}-A_{4} / A_{0}$ & $0.2172279-0.0151979$ \\
$P_{3} / P_{2}-A_{3} / A_{2}$ & $0.6225148-0.3057615$ \\
$P_{4} / P_{3}-A_{4} / A_{3}$ & $0.7627216-0.4759170$ \\
$P_{2} / P_{1}-A_{2} / A_{1}$ & $0.6140875-0.0386031$ \\
$P_{3} / P_{1}-A_{3} / A_{1}$ & $0.3822782-0.0118033$ \\
$P_{4} / P_{1}-A_{4} / A_{1}$ & $0.2915721-0.0056174$ \\
\hline
\end{tabular}

However, no clear sign of period doubling is detected in CoRoT ID 0101368812.

According to their unexpected period ratios $P_{3} / P_{0}=0.285$ and $P_{4} / P_{0}=0.217$, the frequencies $f_{3}$ and $f_{4}$ cannot be explained by any two radial modes and must be interpreted as non-radial modes (Dziembowski \& Smolec 2009). Moreover, when we compare their period ratios with an estimated effective temperature (see Sect. 4) of 6500-7000 K, in Fig. 3 of Kollath et al. (2011), the frequency $f_{3}=7.194 \mathrm{~d}^{-1}$ is located half-way between the sixth and seventh overtones and the frequency $f_{4}=9.433 \mathrm{~d}^{-1}$ is half-way between the eight and ninth overtones, which excludes them from being radial modes.

\section{Fundamental parameters of CoRoT ID 0101368812}

The position of CoRoT ID 0101368812 in the Petersen diagram of Chadid et al. (2010), indicates that it has a very low metallicity and that its observed period ratio of $P_{1} / P_{0}=0.745$ and period of $P_{0}=0.488$ are approximately equal to those of RRd stars. Comparing the period ratio and the fundamental period of CoRoT ID 0101368812 to the results of Szabo et al. (2004), our values match the sequence with $M=0.65-0.71 M_{\odot}$, $L=40-50 L_{\odot}$, and $T_{\text {eff }}=6500-7000 \mathrm{~K}$. The mean surface gravities are also calculated, by using Eq. (15) of Jurcsik (1998), which depends only on the fundamental period (Eq. (2)). We deduce a value of $\log g$ of around 2.855 ,

$\log g=2.473-1.226 \log P$.

Table 2 compares the fundamental parameters of both BS Com and CoRoT ID 0101368812 and indicates a close similarity of our results with those of Dekany et al. (2008) for the RR d star, BS Com, which has a fundamental period (0.4879) and a period ratio $(0.744)$ that are almost equal to those of CoRoT ID 0101368812.

\section{Summary}

Continuous CoRoT photometry of a new CoRoT ID 0101368812 spanning over 152 days, with high time resolution
Table 2. Estimation of fundamental parameters of CoRoT ID 0101368812 and their comparison with those of BS Com of Dekany et al. (2008).

\begin{tabular}{lcc}
\hline \hline & CoRoT ID 0101368812 & BS Com \\
\hline$M / M_{\odot}$ & $0.65-0.71$ & 0.698 \\
$L / L_{\odot}$ & $40-50$ & 51 \\
$Z$ & $0.0001-0.001$ & 0.0006 \\
$T_{\text {eff }}$ & $6500-7000 \mathrm{~K}$ & $6840 \mathrm{~K}$ \\
$\log (g)$ & 2.85 & 2.85 \\
\hline
\end{tabular}

and high photometric precision, provides: (1) an accurate Fourier analysis of a new double mode RR Lyrae star; (2) non-linearly coupled frequencies, and both fundamental and first overtone radial modes $f_{0}$ and $f_{1}$, where the first overtone is the dominant pulsation mode (up to the tenth order); (3) a large number of 22 linear combinations of these modes; (4) additional frequencies $f_{2}, f_{3}$, and $f_{4}$, that have never been seen before in double-mode RR Lyrae stars, which have unexpected period ratios of $P_{2} / P_{0}=0.458$ implying that $f_{2}$ may be either the third radial overtone mode or a non-radial mode, and $P_{3} / P_{0}=0.285$ and $P_{4} / P_{0}=0.217$ implying that $f_{3}$ and $f_{4}$ must be non-radialmodes. The additional frequencies consist of 14 linear combinations with the fundamental and the first overtone radial modes and their harmonics. All the detected coupling terms of positive linear combinations have larger amplitudes than those of negative linear combinations.

These results imply that the newly discovered star CoRoT ID 0101368812 constitutes a new type of RR Lyrae pulsators and its data contributes to theoretical debates on the simultaneous excitation of the non-consecutive multi-overtone radial modes and the non-radial modes in RR Lyrae stars.

Acknowledgements. I thank Lee Murphy for a critical reading of the paper. During this work, I learned of the death of my friend Bernard Reyboz, the sculptor of Moving Objects in Space. This paper is dedicated to his memory.

\section{References}

Antonello, E., \& Aikawa, T. 1998, A\&A, 337, 145 Buchler, J. R., \& Moskalik, P. 1992, ApJ, 391, 736

Chadid, M., Benko, J. M., Szabo, R., et al. 2010, A\&A, 510, A39

Chadid, M., Perini, C., Bono, G., et al. 2011, A\&A, 527, A146

Clement, C. M., Kinman, T. D., \& Suntzeff, N. B. 1991, ApJ, 372, 273

Clementini, G., Di Tomaso, S., Di Fabrizio, L., et al. 2000, AJ, 120, 2054

Dekany, I., Kovacs, G., Jurcsik, J., et al. 2008, MNRAS, 386, 1

Dziembowski, W. A., \& Smolec, R. 2009, AIPC, 1170, 83

Gruberbauer, M., Kolenberg, K., Rowe, J. F., et al. 2007, MNRAS, 379, 1498

Jurcsik, J. 1998, A\&A, 333, 571

Kollath, Z., Molnar, L., \& Szabo, R. 2011, MNRAS, 414, 1111

Lenz, P., \& Breger, M. 2005, CoAst, 146, 53

Moskalik, P., \& Buchler, J. R. 1990, ApJ, 355, 590

Moskalik, P., \& Kolaczkowski, Z. 2008, CoAst, 157, 343

Nemec, J. M. 1984, IAUS, 105, 465

Oaster, L., Smith, H. A., \& Kinemuchi, K. 2006, PASP, 841, 405

Olech, A., \& Moskalik 2009, A\&A, 494, 170

Petersen, J. O. 1973, A\&A, 27, 89

Reegen, P. 2007, A\&A, 467, 1353

Smith, H. A. 1995, RR Lyrae Stars (Cambridge: Cambridge Univ. Press)

Soszynski, I., Poleski, R., Udalski, A., et al. 2008, Acta Astron., 58, 163

Szabo, R., Kollath, Z., \& Buchler, R. 2004, A\&A, 425, 627 
M. Chadid: Detection of multiple modes in a new double-mode RR Lyrae star $(R N)$

Table 3. Data Fourier amplitudes, phases, spectral significance as defined in Reegen (2007), and identification(s) for the CoRoT data of CoRoT ID 0101368812.

\begin{tabular}{ccccc}
\hline \hline $\begin{array}{c}\text { Frequency } \\
{\left[\mathrm{d}^{-1}\right]}\end{array}$ & $\begin{array}{c}\text { Amplitude } \\
{[\mathrm{mag}]}\end{array}$ & $\begin{array}{c}\text { Phase } \\
{[\text { cycles }]}\end{array}$ & Sig & ID \\
\hline
\end{tabular}

$\begin{array}{lllrl}\text { A.1 Fundamental and first overtone frequencies and harmonics } \\ 2.0490089 & 0.05283697 & 0.05393525 & 985.16 & f_{0} \\ 4.0980178 & 0.00549586 & 0.47756543 & 102.47 & 2 f_{0} \\ 6.1476845 & 0.00053213 & 0.17333388 & 9.921 & 3 f_{0} \\ & & & & \\ 2.7502634 & 0.14295027 & 0.01107915 & 2665.35 & f_{1} \\ 5.5003289 & 0.02233101 & 0.08782567 & 416.36 & 2 f_{1} \\ 8.2503071 & 0.00999888 & 0.38163355 & 186.43 & 3 f_{1} \\ 11.000658 & 0.00272524 & 0.71454325 & 50.81 & 4 f_{1} \\ 13.750965 & 0.00118573 & 0.19175027 & 22.10 & 5 f_{1} \\ 16.500329 & 0.00108239 & 0.22729722 & 20.18 & 6 f_{1} \\ 19.250965 & 0.00079608 & 0.69219275 & 14.84 & 7 f_{1} \\ 22.001987 & 0.00026138 & 0.03266887 & 4.87 & 8 f_{1} \\ 24.752281 & 0.00041531 & 0.42704908 & 7.74 & 9 f_{1} \\ 27.502302 & 0.00021500 & 0.52535327 & 4.00 & 10 f_{1}\end{array}$

A.2 Linear combinations of the fundamental and first overtone

$\begin{array}{rllrl}4.7990089 & 0.02375697 & 0.20175714 & 442.95 & f_{1}+f_{0} \\ 6.8483248 & 0.00251834 & 0.82486090 & 46.95 & f_{1}+2 f_{0} \\ 0.7012982 & 0.01723548 & 0.70807264 & 321.36 & f_{1}-f_{0} \\ 1.3470092 & 0.00079826 & 0.34861115 & 14.88 & 2 f_{0}-f_{1} \\ & & & & \\ 7.5493378 & 0.00941423 & 0.46739036 & 175.53 & 2 f_{1}+f_{0} \\ 9.5990045 & 0.00242065 & 0.20063040 & 45.13 & 2 f_{1}+2 f_{0} \\ 11.646040 & 0.00034402 & 0.16590857 & 6.41 & 2 f_{1}+3 f_{0} \\ 3.4509474 & 0.00379389 & 0.87464681 & 70.73 & 2 f_{1}-f_{0} \\ 1.4019386 & 0.00106843 & 0.76426364 & 19.92 & 2 f_{1}-2 f_{0} \\ & & & & \\ 10.299316 & 0.00367972 & 0.90665820 & 68.60 & 3 f_{1}+f_{0} \\ 12.348325 & 0.00093685 & 0.37942015 & 17.46 & 3 f_{1}+2 f_{0} \\ 6.2016271 & 0.00120088 & 0.75258034 & 22.39 & 3 f_{1}-f_{0} \\ & & & & \\ 13.049996 & 0.00141450 & 0.30709406 & 26.37 & 4 f_{1}+f_{0} \\ 15.099005 & 0.00043134 & 0.72425652 & 8.04 & 4 f_{1}+2 f_{0} \\ 8.9516053 & 0.00148625 & 0.98635856 & 27.71 & 4 f_{1}-f_{0} \\ 6.9022675 & 0.00033942 & 0.90457043 & 6.32 & 4 f_{1}-2 f_{0} \\ & & & & \\ 15.799974 & 0.00078626 & 0.62841906 & 14.66 & 5 f_{1}+f_{0} \\ 11.701956 & 0.00078505 & 0.18001103 & 14.63 & 5 f_{1}-f_{0} \\ & & & & \\ 18.549009 & 0.00051792 & 0.61416075 & 9.65 & 6 f_{1}+f_{0} \\ 14.451605 & 0.00041016 & 0.38880583 & 7.64 & 6 f_{1}-f_{0} \\ & & & & \\ 21.299974 & 0.00038183 & 0.21887111 & 7.11 & 7 f_{1}+f_{0} \\ 17.201956 & 0.00026425 & 0.76975610 & 4.92 & 7 f_{1}-f_{0} \\ & & & & \\ 24.050982 & 0.00031356 & 0.63098586 & 5.84 & 8 f_{1}+f_{0} \\ & & & & \\ 22.703601 & 0.00028041 & 0.61825192 & 5.22 & 9 f_{1}-f_{0}\end{array}$

A.3 Additional frequencies

$\begin{array}{rrrrr}4.4786159 & 0.00551832 & 0.08682282 & 102.89 & f_{2} \\ 7.1943909 & 0.00168729 & 0.62087889 & 31.46 & f_{3} \\ 9.4325280 & 0.00080301 & 0.84190927 & 14.97 & f_{4}\end{array}$

A.4 Linear combinations

\begin{tabular}{rrrrr}
7.2276117 & 0.00171597 & 0.91778852 & 31.99 & $f_{1}+f_{2}$ \\
1.7285984 & 0.00120575 & 0.51649016 & 22.48 & $f_{2}-f_{1}$ \\
9.9440402 & 0.00048583 & 0.68722037 & 9.05 & $f_{1}+f_{3}$ \\
\hline
\end{tabular}


A\&A 540, A68 (2012)

Table 3. continued.

\begin{tabular}{cccrl}
\hline \hline $\begin{array}{c}\text { Frequency } \\
{\left[\mathrm{d}^{-1}\right]}\end{array}$ & $\begin{array}{c}\text { Amplitude } \\
{[\mathrm{mag}]}\end{array}$ & $\begin{array}{c}\text { Phase } \\
{[\text { Cycle }]}\end{array}$ & Sig & ID \\
\hline 12.182879 & 0.00070560 & 0.87881138 & 13.15 & $f_{1}+f_{4}$ \\
6.6828788 & 0.00069263 & 0.82350279 & 12.91 & $f_{4}-f_{1}$ \\
& & & & \\
9.9775899 & 0.00040395 & 0.99452770 & 7.53 & $2 f_{1}+f_{2}$ \\
14.932528 & 0.00040083 & 0.04157111 & 7.47 & $2 f_{1}+f_{4}$ \\
& & & & \\
6.5273002 & 0.00100850 & 0.48359169 & 18.80 & $f_{0}+f_{2}$ \\
9.2430709 & 0.00057246 & 0.13788524 & 10.67 & $f_{0}+f_{3}$ \\
11.482195 & 0.00034898 & 0.62818470 & 6.50 & $f_{0}+f_{4}$ \\
& & & & \\
9.2776073 & 0.000644332 & 0.76252971 & 12.01 & $f_{1}+f_{0}+f_{2}$ \\
12.026971 & 0.000399312 & 0.58922521 & 7.44 & $2 f_{1}+f_{0}+f_{2}$ \\
\hline
\end{tabular}

